\title{
The challenge of complexity for cognitive systems
}

\author{
Ute Schmid $^{\mathrm{a}, *}$, Marco Ragni ${ }^{\mathrm{b}}$, Cleotilde Gonzalez ${ }^{\mathrm{c}}$, Joachim Funke ${ }^{\mathrm{d}}$ \\ ${ }^{a}$ Faculty Information Systems and Applied Computer Science, University of Bamberg, 96045 Bamberg, Germany \\ ${ }^{\mathrm{b}}$ Center for Cognitive Science, University of Freiburg, 79098 Freiburg, Germany \\ ${ }^{\mathrm{c}}$ Department of Social \& Decision Sciences, Carnegie-Mellon University, Pittsburgh, PA 15213, USA \\ ${ }^{\mathrm{d}}$ Department of Psychology, University of Heidelberg, 69117 Heidelberg, Germany
}

Available online 28 December 2010

\begin{abstract}
Complex cognition addresses research on (a) high-level cognitive processes - mainly problem solving, reasoning, and decision making - and their interaction with more basic processes such as perception, learning, motivation and emotion and (b) cognitive processes which take place in a complex, typically dynamic, environment. Our focus is on AI systems and cognitive models dealing with complexity and on psychological findings which can inspire or challenge cognitive systems research. In this overview we first motivate why we have to go beyond models for rather simple cognitive processes and reductionist experiments. Afterwards, we give a characterization of complexity from our perspective. We introduce the triad of cognitive science methods - analytical, empirical, and engineering methods - which in our opinion have all to be utilized to tackle complex cognition. Afterwards we highlight three aspects of complex cognition - complex problem solving, dynamic decision making, and learning of concepts, skills and strategies. We conclude with some reflections about and challenges for future research.
\end{abstract}

(C) 2010 Elsevier B.V. All rights reserved.

\section{Introduction}

Dealing with complexity has become one of the great challenges for modern information societies. To reason and decide, plan and act in complex domains is no longer limited to highly specialized professionals in restricted areas such as medical diagnosis, controlling technical processes, or serious game playing. Complexity has reached everyday life and it affects people in such mundane activities such as planning a travel, investing money, or buying a car. The origin of many aspects of everyday complexity is information technology, which made it possible to obtain information about nearly everything at nearly anytime and in any location. For example, now activities such as banking or shopping are independent from location, business time and office

\footnotetext{
* Corresponding author.

E-mail addresses: ute.schmid@uni-bamberg.de (U. Schmid), marco. ragni@uni-freiburg.de (M. Ragni), coty@cmu.edu (C. Gonzalez), funke@ uni-hd.de (J. Funke).
}

hours. While this gives much more autonomy to every person, at the same time, persons are expected to pursue many activities - such as selecting a suitable flight or searching for a suitable book as a present - based on their own judgement by interaction with some computer program instead of getting service and council by a trained person.

In principle, there are two sources of complexity in dealing with information technology: One source of complexity arises from the interaction possibilities offered by the computer and the used software. To provide for cognitively ergonomic interaction technologies is a main topic of research in the domain of human-computer interaction (Dix, Finlay, Abowd, \& Beale, 2004). The other source of complexity arises from the content domain. For example, when deciding whether to invest in stock exchange and, if yes, in which stocks, the internet offers lots of information. Whether someone arrives at a useful decision might depend on his/her background knowledge, search strategy, heuristics and assumptions.

The ultimate hope is that information technology will be enhanced by cognitive systems to support people navigating 
through the jungle of everyday reasoning, decision making, planning and acting by providing intelligent support technology. Lessons learned from expert system research of the 1980s (Hayes-Roth, Waterman, \& Lenat, 1983) are that the aim should not be to provide for fully automated systems which can solve specialized tasks autonomously but instead to develop interactive assistant systems or cognitive companions (Forbus \& Hinrichs, 2006) where user and system work together by taking advantages of the respective strength of human and machine (see Biundo et al., this issue).

However, before we can realize such an intelligent support technology further basic research of complex cognitive systems is needed. Insights in cognitive structures and processes underlying successful human reasoning and planning can provide suggestions for algorithm design. Insights in restrictions, typical errors and misconceptions can provide information about those parts of a complex task from which the human should be relieved. Therefore, research in complex cognition is necessarily an interdisciplinary endeavor where cognitive artificial intelligence $(\mathrm{AI})$ and cognitive psychology work together.

In the following, we will first try to characterize complexity in the context of cognitive systems research. Afterwards, we will give an overview of methodological approaches to tackle complex cognition. Then, we will high-light three central areas of research, namely, complex problem solving, decision making in dynamic and possible real-time environments, and learning high-level strategies for problem solving and reasoning. We will conclude with a final discussion.

\section{Characterizing complex cognitive systems}

In psychological research, cognitive complexity often is used to refer to high-level cognitive processes - mainly problem solving, reasoning, and decision making - and their interaction with more basic processes such as perception, learning, motivation, and emotion (Dörner \& Wearing, 1995; Knauff \& Wolf, 2010; Osman, 2010; Sternberg $\&$ Ben-Zeev, 2001). We propose to use the term complex cognition as a label for a perspective on psychological processes that searches for an integration of various cognitive processes needed for a smart course of action. Complex cognition is opposed to simple cognition, a term that describes isolated capacities of single psychic functions like perception, memory, and thinking without reference to other functions. In a sense, complex cognition tries to address cognition as a whole, whereas simple cognition is interested in the different parts of the psychic system.

As Knauff and Wolf (2010) pointed out, there is a second important aspect to complexity - the complexity given by the environment with which the agent has to interact (Dörner \& Wearing, 1995; Osman, 2010). To discern basic research focussing on simple, highly controlled tasks from research on cognitive processes in complex environments some authors proposed the term macrocognition in contrast to microcognition (Klein et al., 2003).
In the context of AI planning, the complexity of the environment is typically characterized by the following dimensions (Ghallab, Nau, \& Traverso, 2004, chap. 1.6): The number of states might be infinite, due to actions which can create new objects or due to numerical variables. States might be only partially observable and as a consequence, the outcome of actions cannot be predicted with certainty. Another cause for uncertain outcome of actions can be that the environment is nondeterministic due to uncontrollable factors (e.g., gambles), unpredictable changes (e.g., weather), or involvement of other intelligent agents (Wooldridge, 2002). While in a static environment, changes can only be caused by actions of the agent, in dynamic systems, changes in the environment occur over time, which also often results in nondeterminacy from the perspective of the agent (Funke, 2010).

In addition to goals that have to be reached in a final state, there might be more general goals in form of constraints, such as avoiding of damage, or optimization of utility functions which must be taken into consideration. There might be situations where goals cannot be reached by a simple sequence of actions but where conditions, loops, or parallel executions might be necessary. Furthermore, there might be problem environments where it is not enough to consider time only implicitly via state transitions but where the duration of actions or temporal constraints must be taken into account. For example, in driver simulations, an autonomous driver needs to react immediately to dynamic changes such as traffic lights or the unexpected appearance of a pedestrian on the road (Langley \& Choi, 2006). Finally, especially in dynamic, nondeterministic environments, it might be necessary to interleave action planning, action monitoring, and action execution.

About three decades ago, there was some effort to tackle the problem of complexity in cognitive psychology as well as in AI, reflected in psychological research in problem solving, high-level learning and expertise (Anderson, 1987; Chi, Feltovich, \& Glaser, 1981; VanLehn, 1991) and in expert systems research (Hayes-Roth et al., 1983). Since some time, research in both disciplines is focussing on more restricted domains. In cognitive psychology, this is reflected by a dominance of research on the neuro-psychological basis of cognition where experiments typically focus on very simple tasks. In AI this is reflected by a dominance of the development of rigorous methods, e.g. in pattern recognition and statistical machine learning, and efficient algorithms, e.g. in automated planning. While this focus on restricted and isolated problems in both disciplines brought along impressive results, we believe, that the ultimate research goals of cognitive psychology and AI should be kept in mind - that is, to get insight in and technologies for cognition in the real world.

We should give a new look on topics such as reasoning in complex domains, learning from problem solving experience, planning and problem solving in dynamic environments, automated decision making or cognitive assistance systems based on the insights and technological developments gained over last decades. 


\section{Methods to tackle complex cognition}

To get insight into the diverse phenomena of complex cognitive systems, the full spectrum of methods offered by cognitive science should be applied. Cognitive science methods draw from three different research traditions analytical, empirical, and engineering research.

Analytical methods as used in philosophy and linguistics, mathematics and theoretical computer science can help to formalize general regularities as well as general constraints of problem domains. Giving precise insight into problem complexities and offering formal models provide a basis for the design of new intelligent algorithms. For example, Wareham and van Rooij (this issue) define an algorithm-independent computational-level model of analogical generalization and investigate the computational complexity of problems associated with this model. Thereby, they provide insights on conditions under which analogical generalization can be done efficiently. New formal approaches and new algorithms might offer a challenge to existing cognitive systems and trigger their extension and modification to cover additional or more complex cognitive processes. For example, Guhe and collegues (this issue) propose an algebraic approach to concept blending which offers new possibilities for algorithms for discovery and creativity. Schmid and Kitzelmann (this issue) propose inductive programming as an efficient method for learning complex rules from few and only positive examples which might offer the possibility to allow for meta-learning in rule-based systems.

Empirical methods as used in experimental cognitive psychology, in psycho-linguistics, and in neurosciences can help to establish relations between formal and computational models of cognitive systems and human cognitive processes and they also can be used exploratory to give general suggestions about cognitive inspired algorithms. The second way may be called "psychonics" in analogy to bionics where engineering draws new ideas from biological systems (Schmid, 2008). Here the relation to empirical data is rather loose - algorithms are designed such that they cover some human cognitive achievement on the functional level. Many typical cognitive AI systems fall in this category (Forbus \& Hinrichs, 2006; Langley \& Choi, 2006). On the other hand, research in cognitive modeling aims at a more close correspondence between cognitive model and empirical data (Gonzalez, Lerch, \& Lebiere, 2003). Here, the goal is to establish correlations between behavioral data from humans and algorithms for the same tasks, typically comparing relative times and percentages of correct solutions. However, the next most important goal is to be able to predict human behavior in novel conditions, for which the models were not initially built for Gonzalez, Best, Healy, Bourne, and Kole (2010). Investigating complex behavior, empirical data on the behavioral rather than the neuropsychological level usually are more helpful to give rise to develop cognitive systems. However, relating behavioral data to neuropsychological findings might give additional constraints for cognitive models.

Currently, psychological research on complex cognitive phenomena, such as problem solving in complex, dynamical domains are rather rare. Some psychological experiments addressing complex problem solving are presented in this special issue. Osman and Spellman (this issue) investigate human strategies in dealing with complex control tasks. Güss and Dörner (this issue) investigate cultural differences when dealing with a dynamical simulation system.

Besides problem solving, reasoning and decision making are research topics in higher cognition. Research in reasoning is often closely related with specific assumptions about knowledge representation. Some authors argue, that the ability of humans to construct multi-modal representations is crucial to the flexibility of human cognition. While Krumnack et al. (this issue) present experimental work and a cognitive model for relating spatial and verbal reasoning, Kurup et al. (this issue) present a cognitive architecture for integrating multiple representations.

Very often, cognitive models are realized within some cognitive architecture, such as ACT-R (see Möbus et al., this issue; Russwinkel et al., this issue), SOAR (Laird, Newell, \& Rosenbloom, 1987), Icarus (see Choi \& Langley, this issue), Polyscheme (see Kurup et al., this issue). Cognitive architectures offer the advantage that all models share the same general assumptions and constraints which makes it easier to compare concurring models in the same domain. On the other hand, a cognitive architecture restricts the algorithmic possibilities.

While cognitive architectures started with the aim to progress to model complex cognition (Anderson, 1983; Newell, 1990), over the last decade, in cognitive modeling the focus also has been shifted to simpler tasks with the focus on relating cognitive processes to their neuropsychological basis. Moebus et al. (this issue), investigate the brain-mapping hypothesis of ACT-R with more complex problems in the domain of mathematical problem solving. Likewise, Russwinkel et al. (this issue) compare experimental data with an ACT-R to predict errors in mathematical reasoning. A cognitive architecture with focus on modeling highly complex real-world tasks with challenging demands on reaction times is Icarus where, for example, urban driving is modeled (see Choi and Langley, this issue).

Cognitive modeling is not necessarily restricted to the symbolic level. David Marr argued for the idea that science must try to understand information processing systems at three distinct levels. These levels include the computational level (what does the system do), the algorithmic level (how does the system operate, on what representations and how are these manipulated) and finally the implementational level (how is the system physically realized, including neural structures and activities) (Marr, 1982). There is an ongoing research effort to bring both sides nearer together: the subsymbolic level (which is typically associated with the neural implementation of cognitive functions - the 
so-called neural correlates) and the symbolic level. Both sides are necessary, as we know that the brain operates on neural networks and on the other side cognitive science is built upon the idea to model thinking by algorithms (Newell, 1990). Both sides allow for interesting insights: The symbolic part allows to understand the problem and to describe complexity (although from a formal perspective), the neural network levels bring learning into play.

\section{Complex problem solving}

Complex problem solving is the label given to a European research tradition (Frensch \& Funke, 1995) which started in the mid 1970s with work done by Donald Broadbent (e.g., Broadbent, 1977) and Dietrich Dörner (e.g., Dörner, 1980). Their intention was to get complexity into the labs of cognitive psychologists and see how naive subjects (not experts) worked on complex, intransparent, and dynamic problems. The availability of computers helped to realize this idea by allowing to construct complex microworlds (scenarios) which required dynamic decision making - not a single decision act, but a series of decisions dependent from each other and dependent from time.

A complex problem is said to occur when finding the solution demands a series of operations which can be characterized as follows (Dörner, Kreuzig, Reither, \& Stäudel, 1983): The number of elements relevant to the solution process is large (complexity), highly interconnected (connectivity), and the system is dynamically changing over time (dynamics). Neither structure nor dynamics are disclosed to the actor (intransparency). Finally, the goal structure is not as straight forward: in dealing with a complex problem, a person is confronted with a number of different goal facets that have to be weighted and coordinated (polytelic situation).

From the beginning of its use in the mid 1970s, the term "complex problem solving" seemed a bit misleading: It was not the problem solving activity which looked complex (on the contrary: most subjects in the experiments made mistakes and tended to simplify the situation), but the problem situation itself. The famous Lohhausen study (Dörner et al., 1983) reported more than 2000 variables in its system. But from its beginning, the question of situation complexity was a hot topic in the development of the field. For example, Funke (1993) argued for the use of much simpler simulation models based on linear structural models - a line of research which today uses the concept of "minimal complex systems" (Funke, 2010; Greiff, 2011).

Güss and Dörner (this issue) use the dynamic system Coldstore for an analysis of cross-cultural differences in problem solving strategies. Four different successful strategies (adaptive, cautious, switching, or oscillating) show distinctive cultural preferences between Brazil, Germany, United States, India, and the Philippines. The bottom line of their research is that complex cognition (i.e., higher order cognitive processes) depends more on cultural traditions than assumed before.
Osman and Speekenbrink (this issue) used a complex dynamic task environment to demonstrate also strategic differences: depending on the uncertainty that was realized in the system, subjects either varied all cues under unstable conditions or - under stable conditions - took the VOTAT strategy (=vary one thing at a time). The bottom line here is: complex cognition is very sensitive to the predictability of the environment and, thus, sensitive to context.

Choi (this issue) developed the ICARUS architecture to model goal management as part of higher-order cognition. Based on a distinction between conceptual and procedural knowledge, concepts and skills for driving a car in a simulated environment have been modeled. Especially, the simulation was confronted with surprising events like a sudden ambulance vehicle or pedestrians jumping on the street, events that require immediate reaction and a shift in the goal hierarchy.

\section{Decision making in dynamic environments}

Decision-making in dynamic systems requires an ability to deal with incomplete information, highly connected variables and changing environments over time. It has been argued that system thinking is essential to learn to make decisions in dynamic systems (Dörner, 1989; Sterman, 2000; Sterman \& Booth-Sweeney, 2002). For example, Senge (1999) suggested that with systems thinking skills, People learn to better understand interdependency [...], and thereby to deal [...] with the forces that shape the consequences of our actions. Complex dynamic systems are typically defined by highly inter-connected states (cf. Funke, 2006; with intransparency of situations and changes over time, it is hard to trace back changes to one variable or cause, specially when the effects are delayed.

Dynamic Stocks and Flow Systems (DSF) are a special kind of dynamic systems that represent the most essential elements of dynamic systems: stocks, accumulations of a certain amount of a quantifiable unit, and flows, that increase the stock amount (inflow) or decrease it (outflow) over time (Cronin, Gonzalez, \& Sterman, 2009; Dutt \& Gonzales, 2007). These simple systems are dynamic because the system's configuration changes over time. The task of the system agent is to predict the underlying changes and to react accordingly to keep the stock in balance.

Different aspects of dynamic systems have been empirically investigated, from feedback response, time delays, linearity, and non-linearity to reading out minimum and maximum information from graphs representing these changes lead to sub-optimal solutions. Unfortunately, there is increasing and robust evidence of a fundamental lack in the human understanding of accumulation and rates of change; a difficulty called the Stock-Flow (SF) failure (Cronin et al., 2009). The SF failure occurs even in simple problems, such as evaluating the level of water in a bathtub given the amounts of water flowing in (inflow) and out of it (outflow) over time (Sterman \& Booth-Sweeney, 2002). Researchers have used simple problems to ask individuals 
for their basic interpretations of a stock's behavior. For example, researchers often use graphical representations of the inflow and the outflow over time, and ask students to answer questions about the stock or to draw it. Despite the simplicity of these problems, individuals with strong mathematical backgrounds exhibit poor performance; less than $50 \%$ of them answer the stock questions correctly (Cronin \& Gonzalez, 2007).

It has been suggested that people use the "wrong representation" to think about stocks and flows (SF) (Cronin et al., 2009), and students often draw a stock that replicates the pattern of inflow or netflow, while ignoring the effect that both inflow and outflow would have on accumulation over time. These mental procedures that lead to erroneously assume that the stock behaves like the flows was termed the "correlation heuristic" (Cronin et al., 2009). Although the correlation heuristic seems to be robust in SF problems (Cronin \& Gonzalez, 2007; Cronin et al., 2009; Sterman \& Booth-Sweeney, 2002), we know little about the mental procedures people use in solving these problems and why.

Ragni et al. (this issue) investigate the question of how one can best deal (from a formal and cognitive perspective) with dynamic stock and flow systems. They analyze the DSF task proposed by Gonzalez and Dutt, and generalize the task from one to several stocks. They use artificial neural networks (ANNs) for identifying the optimal solution for single stocks, and linear programming with the simplex method for solving the generalized problem. Cognitive models are compared with the Artificial Intelligence model. Their method takes the dynamicity of the DSF task into account: The ANN receives like the participants in the empirical investigations at each time step the new value, which has to be taken into account. So the ANN starts to learn and to adapt at each new function change over time. The neural network used only three hidden layers and took the last three times steps into account similar to a human reasoner who does not only base his decisions at the current time point but takes some previous information into consideration. As there is currently no benchmark available they tested their approach on the functions proposed on the DSF challenge.

Another case for dynamic decision making is the article from Choi (this issue). The ICARUS architecture is applied to model driving a car in a simulated environment (see section Complex problem solving). This case can be seen as an inter-connection between complex problem solving - as dynamicity and time changes play a major role. Different to the described approaches here is that in classical DSF tasks the reasoner has to identify future changes based on current and past data, while an ambulance or a pedestrian crossing the street is not predictable but requires nonetheless an immediate reaction.

\section{Learning to deal with complexity}

The flexibility and adaptiveness of a - natural or artificial - cognitive system depends highly on its ability to learn from previous experience. It is obvious that when dealing with complex environments (as described in the previous sections), it is not possible to rely on a pre-defined fixed set of knowledge and behavioral routines. From the perspective of AI, providing a system which is already equipped with all information necessary to deal successfully with all problems which might arise is impossible due to the knowledge engineering bottleneck (Feigenbaum et al., 1977). From the perspective of cognitive modeling, a completely pre-defined set of knowledge and rules would support a nativistic stance, implying that humans are already born with all the knowledge necessary to deal with the world. Following the standpoint of empiricism, we assume that humans have inborn capabilities for learning from the environment. Furthermore, it is safe to assume that human learning occurs over a hierarchy of levels from very basic sensu-motor coordination over pattern classification to concept learning to the acquisition of skills and strategies.

Machine learning research made considerable progress over the last two decades bringing forward a variety of probabilistic, statistical and neural approaches (Mitchell, 1997). Most approaches are concerned with classification learning where concept learning is subsumed as a special case. Reinforcement learning provides a means to acquire action sequences for interaction with the environment, including an opponent in a game. The progress in machine learning is reflected in recent developments of cognitive architectures which provide mechanism for learning to a far larger degree and with a much larger variety of mechanisms than earlier cognitive architectures.

For example, approaches of Bayesian learning (Cooper \& Herskovits, 1992) recently are applied to model concept acquisition and acquisition of causal knowledge from sparse data as statistical inferences over structured knowledge representations (Tenenbaum, Griffiths, \& Kemp, 2006). In the domain of analogical reasoning and problem solving, over the last years models addressing the acquisition of generalized knowledge structures were proposed (Kuehne, Forbus, Gentner, \& Quinn, 2000; Klenk \& Forbus, 2009; Schwering, Krumnack, Kühnberger, \& Gust, 2009; Weller \& Schmid, 2006). Wareham and van Rooij (this issue) address the conditions under which analogical generalization can be done efficiently.

The cognitive architecture Soar already early on provided a mechanism for learning by chunking simple rules into more complex rules (Laird, Rosenbloom, \& Newell, 1986). Learning is triggered directly in the context of problem solving. Chunking is proposed as a universal learning mechanism that can emulate learning of procedural, episodic and declarative knowledge. A similar procedure, called production compilation is realized in ACT-R (Taatgen \& Anderson, 2002). Most learning processes in ACT-R are realized on a subsymbolic level, that is, production rules are selected by their estimated utility which is modified over experience. Likewise, retrieval from declarative memory depends on history of usage (Anderson et al., 
2004). Soar as well as ACT-R mainly address speed-up effects of learning. Another proposal for modeling skill acquisition based on generalization of goals and chaining of given skills or concepts is realized in the cognitive architecture ICARUS (Langley \& Choi, 2006). A system which takes into account learning of new rules using a rule-extraction-refinement algorithm is Clarion. Clarion (Sun \& Zhang, 2006) is a hybrid cognitive architecture which puts implicit and explicit learning in the focus of cognitive modeling and provides for different learning mechanisms such as backpropagation networks, reinforcement learning and imitative learning.

An approach to high-level learning based on an analytical method to learn generalized recursive rules from sparse, positive experience is presented by Schmid and Kitzelmann (this issue). The authors present a method for learning problem solving strategies, such as solving Tower of Hanoi problems, reasoning rules, such as transitivity of certain relations, recursive concepts, such as ancestor and simple phrase-structure grammars.

\section{Conclusions: about complex cognition}

After we have discussed methods for dealing with complexity and given three research areas as examples for complex cognition, we want to come back to our working definition of complexity (see Section 2) where we distinguished between complexity in the cognitive processes itself and complexity in the environment. A similar characterization of complex cognition is given by Knauff and Wolf (2010, p. 100) in their introduction to a "Special Issue on Complex Cognition" in the journal Cognitive Processing: “As 'complex cognition' we define all mental processes that are used by individuals for deriving new information out of given information, with the intention to solve problems, make decision, and plan actions. The crucial characteristic of 'complex cognition' is that it takes place under complex conditions in which a multitude of cognitive processes interact with one another or with other noncognitive processes."

However, giving a closer look at these characterizations, it becomes obvious that the distinction between simple and complex is rather fuzzy: Distinguishing between complex processes (on the person side) and complex conditions/situations (on the environment side) would allow to construct a two-dimensional space with four cells, simple/complex processes crossed with simple/complex situations. Let us take a look to these four cells by giving potential examples:

(1) simple cognition, simple situation: sitting in an isolated room and hearing a single tone;

(2) complex cognition, simple situation: reading a number and doing very complex things with it (e.g., a mental calculator who extracts the root of a huge number in his mind);

(3) simple cognition, complex situation: a manager reads a single figure out of a table within a monthly report of operations;
(4) complex cognition, complex situation: a busy manager makes a series of interdependent decisions in a dynamic and complex economic environment.

What is the scale of complex cognition? The four cases illustrate the difficulty of drawing a demarcation line between simple and complex - if you go into detail, previously simple processes can become very complex: Even the simple case of hearing a tone might become a very complex activity if we draw attention to the neural activities needed in this case. On the other hand, even highly complex decisions in highly complex situations can (sometimes) be taken by very simple heuristics (Marewski, Gaissmaier, \& Gigerenzer, 2010).

What possibilities do we have for making decisions about the simple/complex process dimension? Here are some candidates: (a) The number of assumed cognitive processes, (b) the diversity of involved processes, (c) the time taken for processing, (d) the complexity of the simplest machine program which would solve the task.

To assess the complexity of situations, there is also no simple solution. There are no clear measures for system complexity (Kolmogorov complexity and other quantitative measures from mathematics seem to us not appropriate), network theory from physics (Buchanan, 2002; Newman, 2003) might be a starting point.

Until we have no clear indicators, our decisions about simple or complex remain totally subjective and probably unjustly. But for now, we have to live with this uncomfortable situation. Nevertheless, it is our strong belief that there is a need to go beyond today's reductionist psychological experiments and to try hard to push cognitive modeling towards dealing with more and more complex phenomena of human achievements. The special issues dedicated to complex cognition in Cognitive Processing and in Cognitive Systems Research are first evidence that both cognitive psychologists as well as researchers in cognitive AI are trying to move from simple to complex.

\section{Acknowledgements}

We thank the team of reviewers for their constructive comments and suggestion to the authors of the special issue on complex cognition: Ruth Byrne (Trinity College Dublin, University of Dublin, Ireland), Claus-Christian Carbon (University of Bamberg, Germany), Nicholas Cassimatis (Rensselaer Polytechnic Institute, USA), Varun Dutt (Carnegie Mellon University, USA), Christian Freksa (University of Bremen, Germany), Ken Forbus (Northwestern University, USA), Hector Geffner (Universitat Pompeu Fabra, Spain), Helmar Gust (University of Osnabrück, Germany), Joachim Hertzberg (University of Osnabrück, Germany), Krystof Janowicz (Pennsylvania State University, USA), Ion Juvina (Carnegie Mellon University, USA), Matthew Klenk (Naval Research Laboratory, USA), Guenther Knoblich (Radboud University Nijmegen, The Netherlands), Pieter Koopmann (University of 
Nijmegen, The Netherlands), Emiel Kramer (Tilburg University, The Netherlands), Josef Krems (University of Chemnitz, Germany), Antje Krumnak (University of Giessen, Germany), Kai-Uwe Kühnberger (University of Osnabrück, Germany), Pat Langley (Arizona State University, USA), Christian Lebiere (Carnegie Mellon University, USA), Claus Möbus (University of Oldenburg, Germany), Magda Osman (University College London, UK), David Reitter (Carnegie Mellon University, USA), Jussi Rintanen (NICTA, Australia), Frank Ritter (Pennsylvania State University, USA), Klaus Robering (University of Southern Denmark, Denmark), Christoph Schlieder (University of Bamberg, Germany), Wolfgang Schoppek (University Bayreuth, Germany), Gerhard Strube (University of Freiburg, Germany), Niels Taatgen (University of Groningen, The Netherlands), Manfred Thüring (Technical University Berlin, Germany), Elke van der Meer (Humboldt University Berlin, Germany), Walter Warwick (Alion Science and Technology, USA).

\section{References}

Anderson, J. (1987). Skill acquisition: Compilation of weak-method problem solutions. Psychological Review, 94, 192-210.

Anderson, J., D, B., Byrne, M., Douglass, S., Lebiere, C., \& Qin, Y. (2004). An integrated theory of the mind. Psychological Review, 111(4), 1036-1060.

Anderson, J. R. (1983). The architecture of cognition. Cambridge, MA: Harvard University Press.

Broadbent, D. E. (1977). Levels, hierarchies, and the locus of control. Quarterly Journal of Experimental Psychology, 29, 181-201.

Buchanan, M. (2002). Nexus: Small worlds and the groundbreaking theory of networks. New York: Norton.

Chi, M., Feltovich, P., \& Glaser, R. (1981). Categorization and representation of physics problems by experts and novices. Cognitive Science, 5 , 121-152.

Cooper, G., \& Herskovits, E. (1992). A bayesian method for the induction of probabilistic networks from data. Machine Learning, 9, 309-347.

Cronin, M., \& Gonzalez, C. (2007). Understanding the building blocks of system dynamics. System Dynamics Review, 23(1), 1-17.

Cronin, M., Gonzalez, C., \& Sterman, J. D. (2009). Why don't welleducated adults understand accumulation? A challenge to researchers, educators and citizens. Organizational Behavior and Human Decision Processes, 108, 116-130.

Dix, A., Finlay, J., Abowd, G., \& Beale, R. (2004). Human-computer interaction (3rd ed.). New Jersey: Prentice Hall.

Dörner, D. (1980). On the difficulty people have in dealing with complexity. Simulation \& Games, 11, 87-106.

Dörner, D. (1989). Die Logik des Misslingens. Strategisches Denken in komplexen Situationen. Reinbek bei Hamburg: Rowohlt.

Dörner, D., Kreuzig, H. W., Reither, F., \& Stäudel, T. (1983). Lohhausen. vom umgang mit unbestimmtheit und komplexität. Bern: Huber.

Dörner, D., \& Wearing, A. (1995). Complex problem solving: Toward a (computer-simulated) theory. In P. Frensch \& J. Funke (Eds.), Complex problem solving: The european perspective (pp. 65-99). Hillsdale, NJ: Lawrence Erlbaum.

Dutt, V., \& Gonzales, C. (2007). Slope of inflow impacts dynamic decision making. In Proceedings of the 25th International Conference of the System Dynamics Society (pp. 79). Boston, MA: System Dynamics Society.

Feigenbaum, E. (1977). The art of artificial intelligence: themes and case studies in knowledge engineering. In Proceedings of the 5th international joint conference on artificial intelligence (Vol. 2, pp. 1014-1029).
Forbus, K. D., \& Hinrichs, T. R. (2006). Companion cognitive systems A step toward human-level AI. AI Magazine, Achieving Human-Level Intelligence through Integrated Systems and Research, 27(2), 83-95, special issue.

Frensch, P. A., \& Funke, J. (Eds.). (1995). Complex problem solving: The European perspective. Hillsdale, NJ: Lawrence Erlbaum Associates.

Funke, J. (1993). Microworlds based on linear equation systems: A new approach to complex problem solving and experimental results. In G. Strube \& K.-F. Wender (Eds.), The cognitive psychology of knowledge. Amsterdam: Elsevier, pp. 313-330.

Funke, J. (2006). Komplexes problemlösen. In J. Funke (Ed.). Denken und problemlösen (Vol. 8). Göttingen: Hogrefe.

Funke, J. (2010). Complex problem solving: A case for complex cognition? Cognitive Processing, 11(2), 133-142.

Ghallab, M., Nau, D., \& Traverso, P. (2004). Automated planning: Theory and practice. San Francisco, CA: Morgan Kaufman.

Gonzalez, C., Best, B., Healy, A., Bourne, L., \& Kole, J. (2010). A cognitive modeling account of fatigue. Journal of Cognitive Systems Research, 12, 19-32.

Gonzalez, C., Lerch, F. J., \& Lebiere, C. (2003). Instance-based learning in dynamic decision making. Cognitive Science, 27, 591-635.

Greiff, S. (2011). Individualdiagnostik der komplexen Problemlösefähigkeit. Münster: Waxmann.

Hayes-Roth, F., Waterman, D., \& Lenat, D. B. (Eds.). (1983). Building expert systems. Menlo Park, CA: Addison-Wesley.

Klein, G., Ross, K. G., Moon, B. M., Klein, D. E., Hoffman, R. R., \& Hollnagel, E. (2003). Macrocognition. IEEE Intelligent Systems, 18(3), $81-85$.

Klenk, M., \& Forbus, K. (2009). Analogical model formulation for transfer learning in ap physics. Artificial Intelligence, 173(18), 1615-1638.

Knauff, M., \& Wolf, A. G. (2010). Complex cognition: The science of human reasoning, problem-solving, and decision-making. Cognitive Processing, 11(2), 99-102.

Kuehne, S., Forbus, K., Gentner, D., \& Quinn, B. (2000). SEQL: Category learning as progressive abstraction using structure mapping. In L. Gleitman \& A. Joshi (Eds.), Proc. of the 22nd annual conference of the cognitive science society. Mahwah, NJ: Erlbaum, pp. 770775

Laird, J. E., Newell, A., \& Rosenbloom, P. S. (1987). Soar: An architecture for general intelligence. Artificial Intelligence, 33, 1-64.

Laird, J. E., Rosenbloom, P. S., \& Newell, A. (1986). Chunking in soar: The anatomy of a general learning mechanism. Machine Learning, 1, $11-46$.

Langley, P., \& Choi, D. (2006). A unified cognitive architecture for physical agents. In Proceedings of the twenty-first national conference on artificial intelligence. Boston, MA: AAAI Press.

Marewski, J., Gaissmaier, W., \& Gigerenzer, G. (2010). Good judgements do not require complex cognition. Cognitive Processing, 11(2), $103-122$.

Marr, D. (1982). Vision. Cambridge, MA: MIT Press.

Mitchell, T. M. (1997). Machine learning. New York: McGraw-Hill.

Newell, A. (1990). Unified theories of cognition. Cambridge, MA: Harvard University Press.

Newman, M. E. J. (2003). The structure and function of complex networks. SIAM Review, 45, 167-256.

Osman, M. (2010). Controlling uncertainty: A review of human behavior in complex dynamic environments. Psychological Bulletin, 136(1), 65-86.

Schmid, U. (2008). Cognition and AI. Künstliche Intelligenz, 22(1), 5-7.

Schwering, A., Krumnack, U., Kühnberger, K.-U., \& Gust, H. (2009). Syntactic principles of heuristic-driven theory projection. Cognitive Science Research, 10, 251-269.

Senge, P. e. a. (1999). The dance of change: The challenges to sustaining momentum in learning organizations. New York: Doubleday.

Sterman, J. (2000). Business dynamics: System thinking and modeling for a complex world. New York: Irwin/McGraw-Hill. 
Sterman, J., \& Booth-Sweeney, L. (2002). Cloudy skies: Assessing public understanding of global warming. System Dynamics Review, 18(2), 207-240.

Sternberg, R., \& Ben-Zeev, T. (2001). Complex cognition: The psychology of human thought. New York: Oxford University Press.

Sun, R., \& Zhang, X. (2006). Accounting for a variety of reasoning data within a cognitive architecture. Journal of Experimental and Theoretical Artificial Intelligence, 18, 169-191.

Taatgen, N. A., \& Anderson, J. R. (2002). Why do children learn to say 'broke'? A model of learning the past tense without feedback. Cognition, 86, 123-155.
Tenenbaum, J., Griffiths, T., \& Kemp, C. (2006). Theory-based Bayesian models of inductive learning and reasoning. Trends in Cognitive Sciences, 10(7), 309-318.

VanLehn, K. (Ed.). (1991). Architectures for intelligence. Hillsdale, NJ: Lawrence Erlbaum.

Weller, S., \& Schmid, U. (2006). Solving proportional analogies by Egeneralization. In KI 2006: Advances in artificial intelligence, 29th annual German conference on AI, KI 2006, proceedings. Heidelberg: Springer.

Wooldridge, M. (2002). An introduction to multiagent systems. New York: Wiley. 